\title{
Evaluación de la reacción acrosomal en espermatozoides humanos inducida por los monosacáridos manosa y N-acetilglucosamina
}

\author{
W.D. Cardona-Maya, A.P. Cadavid \\ Grupo Reproducción-Biogénesis, Universidad de Antioquia, Medellín, Colombia.
}

Actas Urol Esp 2005; 29 (7) 676-684

\section{RESUMEN}

EVALUACIÓN DE LA REACCIÓN ACROSOMAL EN ESPERMATOZOIDES HUMANOS INDUCIDA POR LOS MONOSACÁRIDOS MANOSA Y N-ACETILGLUCOSAMINA

Objetivo: El objetivo de esta investigación fue evaluar el papel de los monosacáridos, manosa y Nacetilglucosamina en la inducción de la reacción acrosomal en espermatozoides humanos.

Método: Cada individuo donó una muestra de semen, la cual fue sometida al proceso de capacitación usando albúmina sérica bovina, posterior a lo cual se evaluó el porcentaje de espermatozoides que presentaban reacción acrosomal al estimularlos con manosa y $\mathrm{N}$-acetilglucosamina, usando microscopía de fluorescencia y citometría de flujo.

Resultados: Los resultados obtenidos en las 10 muestras evaluadas mediante citometría de flujo mostraron que sólo el ionóforo de calcio A23187 indujo reacción acrosomal ( $<<0,001)$; en contraste por microscopía de fluorescencia usando la lectina PSA-FITC, se observó un aumento estadísticamente significativo en la reacción acrosomal inducida con manosa y $\mathrm{N}$-acetilglucosamina $(\mathrm{p}<0,001)$.

Conclusiones: Los monosacáridos manosa y $\mathrm{N}$-acetilglucosamina inducen reacción acrosomal parcial y no completa, por lo tanto el espermatozoide gracias a la reacción acrosomal parcial y a las modificaciones morfológicas que sufre durante ese proceso es capaz de fertilizar el oocito.

Palabras Clave: Espermatozoide. Cocito. Receptor. Zona pelúcida. Reacción acrosomal.

\section{ABSTRACT \\ EVALUATION OF THE ROLE OF THE MONOSACCHARIDES, MANNOSE AND N-ACETYLGLUCOSAMINE IN THE INDUCTION OF THE ACROSOME REACTION IN HUMAN SPERMATOZOA}

Objective: The aim of this investigation was to evaluate the role of the monosaccharides, mannose and $\mathrm{N}$-acetylglucosamine in the induction of the acrosome reaction in human spermatozoa.

Methods: Each individual gave a semen sample, the samples were capacitated using bovien serum albumin; the analysis of the mannose and $\mathrm{N}$-acetylglucosamine induced acrosome reaction was evaluated for fluorescence microscopy and flow cytometry.

Results: The results obtained in the acrosomal reaction in the 10 samples evaluated by flow cytometry showed that only calcium ionophore A23187 induced acrosomal reaction $(\mathrm{p}<0.001)$; in contrast, by fluorescence microscopy, was observed a statistically significant increase in the induced acrosomal reaction with mannose and $\mathrm{N}$-acetylglucosamine $(\mathrm{p}<0.001)$.

Conclusions: The monosaccharides mannose and $\mathrm{N}$-acetylglucosamine induces partial acrosomal reaction and it does not complete, therefore the spermatozoa due to partial acrosomal reaction and the morphologics modifications that suffer during that process is able to fertilize the oocyte.

Keywords: Spermatozoa. Cocyte. Receptor. Zona pellucida. Acrosome reaction. 
$\mathrm{L}$ a reacción acrosomal es un proceso especializado de fusión de la membrana citoplasmática con la membrana acrosomal externa en la zona apical de la cabeza espermática originando la liberación de las enzimas almacenadas en el acrosoma y la exposición de la membrana acrosomal interna. La importancia de la reacción acrosomal podría ser la liberación de las enzimas hidroliticas que son requeridas para que el espermatozoide pueda penetrar la zona pelúcida; este proceso se desencadena luego que el espermatozoide entra en contacto con una serie de moléculas presentes en la zona pelúcida ${ }^{1}$, lo cual permite el reconocimiento específico entre el espermatozoide y el oocito.

Para que ocurra la reacción acrosomal y la fertilización, se requiere que el espermatozoide sufra un proceso de capacitación, que se da durante el paso a través del tracto reproductor femenino donde ocurre la interacción entre los espermatozoides y las células del epitelio oviductal $^{2}$. Es aceptado que estos dos eventos, capacitación y reacción acrosomal, están unidos secuencial y funcionalmente y que varios de los procesos de activación de señales intracelulares que ocurren durante la reacción acrosomal, fueron iniciados durante la capacitación.

La zona pelúcida es sintetizada por los oocitos en los roedores y por las células de la granulosa en cerdos, vacunos y humanos. En los murinos, la zona pelúcida está compuesta por 3 glicoproteínas sulfatadas ZP1 (185-200 KDa), ZP2 (120$140 \mathrm{KDa})$ y ZP3 (83 KDa); en los humanos tiene las mismas glicoproteínas pero de menor tamaño, 90-110, 64-76 y 57-73 KDa, respectivamente. Mediante microscopía electrónica se observa que en ambos modelos, murino y humano, la zona pelúcida es una matriz homogénea de filamentos interconectados ${ }^{3}$ que en su estructura y composición forman una red tridimensional decorada por una compleja mezcla de cadenas de oligosacáridos $^{2}$.

Una gran variedad de moléculas han sido propuestas como receptores candidatos de la zona pelúcida en la superficie espermática; entre estos receptores se pueden mencionar: la tirosina quina$s a^{4,5}$, la $\beta-1,4$ galactosiltransferasa ${ }^{6,7}$, el Sp-56 $6^{8}$, las espermadesinas ${ }^{9}$ y las zonadesinas ${ }^{10,11}$. Estas moléculas han sido evaluadas en diferentes espe- cies y ninguna ha podido ser reconocida como el receptor único que permite la interacción entre el espermatozoide y la zona pelúcida.

Los primeros eventos que ocurren durante la fertilización pueden ser vistos como una forma altamente compleja de reconocimiento célula a célula, que involucra cadenas de carbohidratos en las membranas ${ }^{12-14}$. Algunas observaciones han mostrado que los carbohidratos están involucrados en varios pasos durante la fertilización pero particularmente en la unión del espermatozoide a la zona pelúcida ${ }^{15-22}$, en la reacción acrosomal ${ }^{21,23-25}$ y en la fusión espermatozoide-oocito ${ }^{26}$.

La participación de los residuos de carbohidratos en la unión entre el espermatozoide y el oocito en humanos ha sido ampliamente estudiada ${ }^{19,21}$; por ejemplo, la preincubación de espermatozoides con manosa y fucosa altera la capacidad de unión de estas células a la zona pelúcida, debido al bloqueo de los receptores espermáti$\cos ^{19,21,27-29}$. El receptor de manosa, en particular, participa en la interacción intergametos y su distribución topográfica en espermatozoides humanos está fuertemente correlacionada con el estado del acrosoma; luego que el espermatozoide sufre la reacción acrosomal el receptor de manosa se desplaza a la región ecuatorial simultáneamente con la liberación del contenido del acrosoma para permitir la fusión de la membrana plasmática del espermatozoide con la membrana plasmática del oocito ${ }^{30,31}$. Los niveles de colesterol en la membrana plasmática del espermatozoide regulan la aparición de receptores de manosa debido a que, durante el proceso de capacitación, existe una salida de colesterol por la presencia de moléculas aceptoras de colesterol como la albúmina sérica bovina, creando un estado termodinámico favorable para la salida de los receptores de manosa, desde almacenamientos internos, a la membrana plasmática exter$\mathrm{na}^{30}$.

La N-acetilglucosamina, por su parte, es otro carbohidrato que también participa en la interacción intergametos en humanos. Hace algunos años se reportó que la $\mathrm{N}$-acetilglucosamina inducía la reacción acrosomal en espermatozoides humanos a través de mecanismos similares a los descritos en la inducción de esta reacción por la 
zona pelúcida, además se correlacionó con la fertilización in vitro ${ }^{23,32}$. Estos resultados sugieren que la reacción acrosomal inducida por la N-acetilglucosamina y por la zona pelúcida comparten muchas características. La participación de los residuos de $\mathrm{N}$-acetilglucosamina de la zona pelúcida en la interacción espermatozoide-oocito en humanos podría ser sustentada en ensayos donde se trataron los oocitos con $\mathrm{N}$-acetilglucosaminidasas y se bloqueó la capacidad de unión de los espermatozoides ${ }^{25}$, lo que sugiere la presencia de sitios de unión de $\mathrm{N}$-acetilglucosamina en el espermatozoide.

Generalmente los oligosacáridos que han sido evaluados para la inducción de la reacción acrosomal están unidos a albúmina sérica bovina (BSA) con el fin de formar una estructura tridimensional, sin embargo surge la duda de si realmente el efecto inductor lo está realizando el oligosacárido o la proteína ${ }^{16,18,33,34}$. El objetivo de este estudio fue evaluar el efecto de los monosacáridos manosa y $\mathrm{N}$-acetilglucosamina, libres de $\mathrm{BSA}$, en la inducción de la reacción acrosomal en espermatozoides humanos capacitados.

\section{METODOLOGÍA}

Muestras de semen

Se analizaron muestras de semen de 10 individuos que fueron tomadas mediante masturbación en un recipiente estéril, posterior a una abstinencia de 2 a 5 días. Todos los individuos tenian sus parámetros seminales normales conrespecto a la $\mathrm{OMS}^{36}$.

\section{Separación y capacitación espermática}

La separación y la capacitación espermática se realizaron simultáneamente variando el protocolo descrito por Jondet ${ }^{35}$. Brevemente, los espermatozoides fueron incubados durante períodos de 5 ó 12 horas a $37^{\circ} \mathrm{C} / 5 \% \mathrm{CO}_{2}$ en un tubo de Jondet con medio HAM F-12 suplementado con $35 \mathrm{mg} / \mathrm{ml} \mathrm{de}$ albúmina sérica bovina -BSA- (Sigma Chemical Company, St Louis, MO, USA); luego de la incubación se recuperaron los espermatozoides capacitados del compartimiento interno de dicho tubo.

\section{Inducción de la reacción acrosomal}

La inducción de la reacción acrosomal se realizó estimulando los espermatozoides con 10 y $100 \mathrm{mg} / \mathrm{ml}$ de los monosacáridos, manosa y $\mathrm{N}$-acetilglucosamina (Sigma Chemical Company, St Louis, MO, USA) y $10 \mu \mathrm{M}$ de ionóforo de calcio A23187 (Sigma Chemical Company, St Louis, MO, USA), durante 3 horas a $37^{\circ} \mathrm{C} / 5 \% \mathrm{CO}_{2}$.

\section{Evaluación de la reacción acrosomal por} microscopía de fluorescencia

Aproximadamente $1 \times 10^{6}$ espermatozoides fueron lavados dos veces en buffer fosfato salino (PBS), depositados sobre portaobjetos, fijados por exposición a metanol frío/30 segundos y coloreados 30 minutos/cámara húmeda con la lectina Pisum sativum aglutinina marcada con isotiocianato de fluoresceína -PSA-FITC- (Sigma Chemical Company, St Louis, MO, USA) a una concentración de $50 \mu \mathrm{g} / \mathrm{ml}$ en PBS. Luego de dos lavados con PBS, los extendidos secos fueron montados en glicerol al 90\% en PBS y se evaluaron 100 espermatozoides en el microscopio de fluorescencia a una longitud de onda de $485 \mathrm{~nm}$ con un aumento de 100X, para determinar el porcentaje de espermatozoides reaccionados. Se consideraron como espermatozoides reaccionados aquellos que presentaban fluorescencia sólo en la región ecuatorial y espermatozoides intactos, aquellos que presentaron todo el acrosoma fluorescente, (Fig. 1).

\section{Evaluación de la reacción acrosomal por citometría de flujo}

Una suspensión de $1 \times 10^{6}$ espermatozoides se incubó con $10 \mu \mathrm{l}$ de IgG humana (Miltenyi Bistec GMBH, Bergisch Gladbach, Germany) durante $30 \mathrm{~min} / \mathrm{T}^{\mathrm{o}} \mathrm{amb}$ para bloquear la unión a los receptores Fc. Después de dos lavados con PBS a 600rpm/8 minutos, se agregaron $10 \mu \mathrm{ldel}$ anticuerpo IgG de ratón contra CD46 humano conjugado con FITC (BD PharMingen San Diego, USA); en otra alícuota se agregó un control de isotipo utilizando un anticuerpo IgG de ratón contra trinitrofenol (BD PharMingen San Diego, USA). Las muestras se incubaron a temperatura ambiente protegidas de la luz, se lavaron con PBS y luego se les adicionó $2,5 \mu \mathrm{g} / \mathrm{ml}$ de ioduro de propidio (Sigma Chemical Company, St Louis, MO, USA) para evaluar la viabilidad espermática. Se realizó una adquisición de 10.000 eventos usando un citómetro de flujo Epics XL (Coulter) y se determinó el porcentaje de células reaccionadas con el programa WinMDi $2,8^{\circledR}$. 


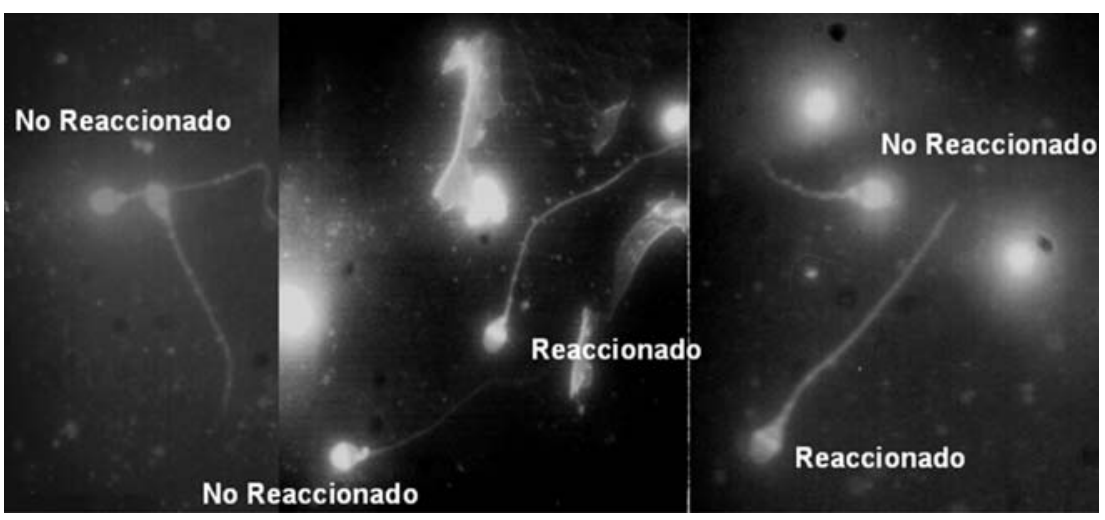

FIGURA 1. Patrón de espermatozoides reaccionados y no reaccionados coloreados con la lectina PSA marcada con isotiocianato de fluoresceina.

ellos los parámetros seminales son normales según la $\mathrm{OMS}^{36}$. Todos los individuos eran aparentemente sanos, con fertilidad desconocida y tenían una edad promedio de 24,8 años (21-32).

Estandarización de la inducción de la reacción acrosomal

Para determinar el mejor tiempo en el cual se evaluaría la reacción acrosomal, se realizó una cinética de inducción de la reacción acrosomal con $10 \mathrm{mg} / \mathrm{ml}$ de monosa-

\section{Análisis estadístico}

La comparación estadística entre los estímulos evaluados se realizó con una prueba de Anova no paramétrica (Kruskal-Wallis); para obtener información adicional sobre el comportamiento de los grupos estimulados a las diferentes concentraciones de los monosacáridos, se realizó la prueba de comparación múltiple de Dunn's; para corroborar que existiera diferencia entre el control positivo con ionofóro de calcio A23187 y la reacción acrosomal espontánea se uso la prueba de Mann-Whitney. Todos los análisis se hicieron con el paquete estadístico Prism ${ }^{\circledR}$ version 3.02 Software.

\section{RESULTADOS}

Descripción de los eyaculados

En la Tabla 1 se hace una descripción de las características de los eyaculados de los 10 individuos analizados y se observa que en todos

Tabla 1

Descripción de los parámetros seminales analizados de cada uno de los individuos, comparados frente al valor de referencia de la Organización Mundial de la Salud de $1999^{36}$.

\begin{tabular}{lcc}
\hline & $\begin{array}{c}\text { Valor de los } \\
\text { 10 individuos }\end{array}$ & Valor OMS $^{\mathbf{3 6}}$ \\
\hline Volumen (ml) & $3,7(2.2-5.0)$ & $\geq 2.0$ \\
Viabilidad (\%) & $83,8(79-88)$ & $>50$ \\
Morfología (\%) & $17,8(16-20)$ & $>15$ \\
Concentración pre-capacitación $\left(10^{6} / \mathrm{ml}\right)$ & $50(25-86)$ & $\geq 20$ \\
Movilidad (a+b) pre-capacitación $(\%)$ & $60,6(54-75)$ & $>50$ \\
\hline
\end{tabular}
cáridos, con tiempos desde 0 hasta 240 minutos. Los resultados usando tanto citometría de flujo como microscopía de fluorescencia mostraron que el tiempo óptimo para la valoración del porcentaje de inducción de reacción acrosomal por los monosacáridos fue de 180 minutos postinducción (Fig. 2).

\section{Evaluación de la reacción acrosomal}

Los resultados obtenidos en las 10 muestras evaluadas mediante citometría de flujo mostraron que sólo el ionóforo de calcio A23187 indujo reacción acrosomal $(\mathrm{p}<0,001)$. En contraste, mediante la evaluación de la reacción acrosomal por microscopía de fluorescencia usando la lectina PSA-FITC, se observó un aumento estadísticamente significativo en la reacción acrosomal inducida con manosa y $\mathrm{N}$-acetilglucosamina a las concentraciones de 10 y $100 \mathrm{mg} / \mathrm{ml} \quad(\mathrm{p}<0,001)$ (Fig. 3)

Aunque el porcentaje de reacción acrosomal varió de donante a donante, en la Figura 4 se ilustran los resultados típicos obtenidos mediante citometría de flujo: en FL1 las células marcadas con el anticuerpo antiCD46-FITC y en FL2 las células que adquirieron o no el ioduro de propidio. Para intentar explicar los resultados contradictorios de la reacción acrosomal inducida con los monosacáridos por las técnicas de microscopía de fluorescencia y de citometría de flujo, se realizó un análisis de las células vivas y las células muertas en los 


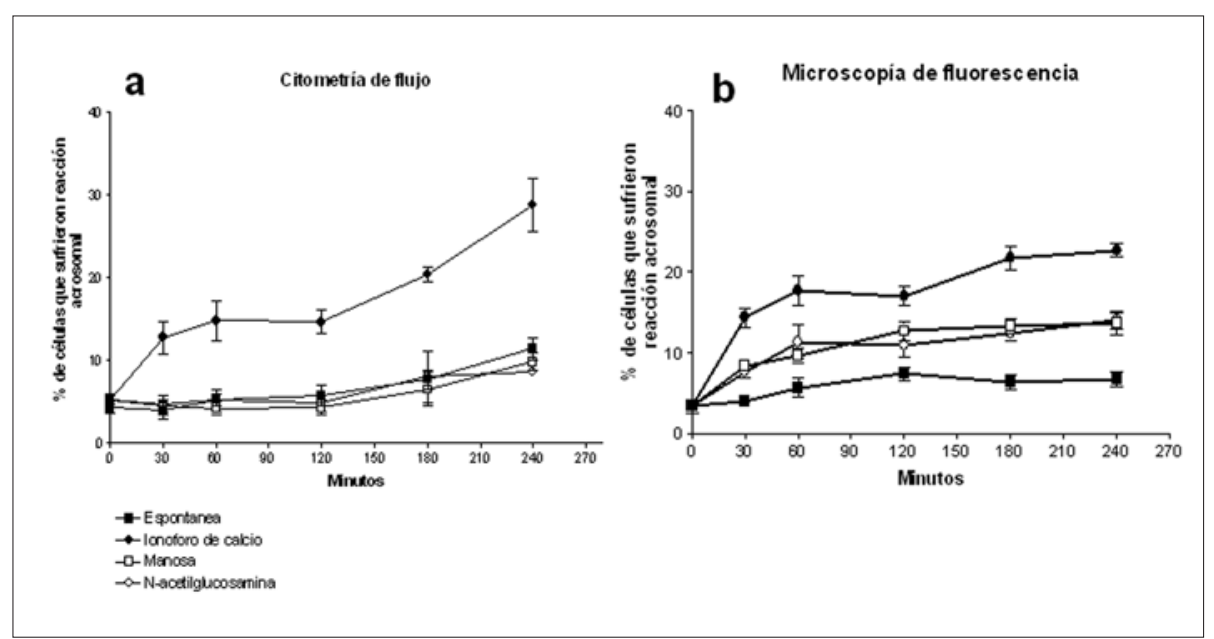

FIGURA 2. Cinética de la inducción de la reacción acrosomal por las técnicas de citometria de flujo (a) y microscopia de fluorescencia (b).

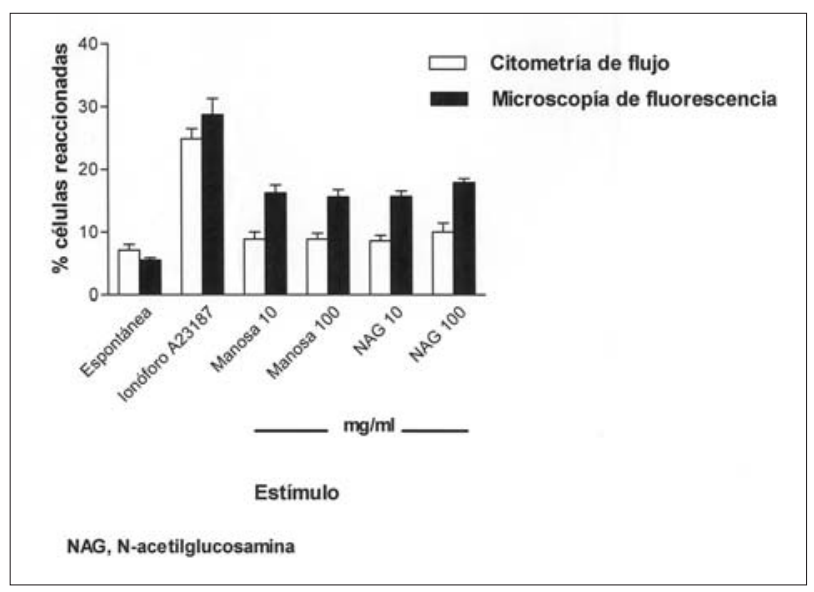

FIGURA 3. Efecto de la inducción de la reacción acrosomal inducida por los monosacárido, manosa y N-acetilglucosamina, evaluada por citometría de flujo y por microscopia de fluorescencia.

datos de la citometría de flujo, diferenciando la incorporación o no del ioduro de propidio y no se encontró diferencias en los valores obtenidos agrupando las células vivas y las muertas o analizándolos por separado. Sin embargo es importante tener en cuenta que las muestras de semen usadas para la inducción de la reacción acrosomal en cada uno de los análisis presentaron altas proporciones de células vivas $(>80 \%$ ).

\section{Efecto del tiempo de capacitación en la reacción acrosomal}

Se evaluó la diferencia en el proceso de capacitación durante 5 y 12 horas con medio HAM F12 suplementado con $35 \mathrm{mg} / \mathrm{ml}$ de BSA y no se observó diferencia estadísticamente significativa en el porcentaje de células que presentaban reacción acrosomal en los dos tiempos, tanto por citometría de flujo como por microscopía de fluorescencia (Fig. 5); la distribución de los datos en ambos grupos fue similar.

\section{DISCUSIÓN}

En el presente estudio se evaluó el papel de los monosacáridos manosa y $\mathrm{N}$-acetilglucosamina en la inducción de la reacción acrosomal en espermatozoides humanos, posterior a la capacitación. In vivo, los espermatozoides migran hacia el encuentro del oocito después de su tránsito a través del tracto reproductor femenino, donde se lleva a cabo el proceso de capacitación y estos espermatozoides capacitados son los que pueden penetrar la zona pelúcida y fertilizar el oocito. En nuestro sistema de laboratorio, los espermatozoides eyaculados fueron capacitados incubando los espermatozoides en un tubo de Jondet ${ }^{35,37}$ con medio HAM-F12 suplementado con BSA. Durante esta incubación con la albúmina, se retira el colesterol de la membrana plasmática del espermatozoide debido a la capacidad de la albúmina de aceptar moléculas de colesterol, lo que produce un incremento de la fluidez, una remoción de componentes y un reordenamiento de las proteínas de la membrana. Uno de los cambios más importantes es la salida de los receptores de manosa de almacenamientos internos $^{30}$ que podría explicar el aumento en el número de células que presentaron reacción acrosomal por microscopía de fluorescencia después de la inducción con $10 \mathrm{mg} / \mathrm{ml}$ de manosa (Fig. 2).

Los resultados obtenidos mediante microscopía de fluorescencia después de la inducción de la reacción acrosomal (Fig. 3), muestran que el ionóforo de calcio utilizado como control positivo, produce aproximadamente el doble de inducción de reacción acrosomal que los estímulos, lo cual nos permite proponer que no sólo los monosacá- 


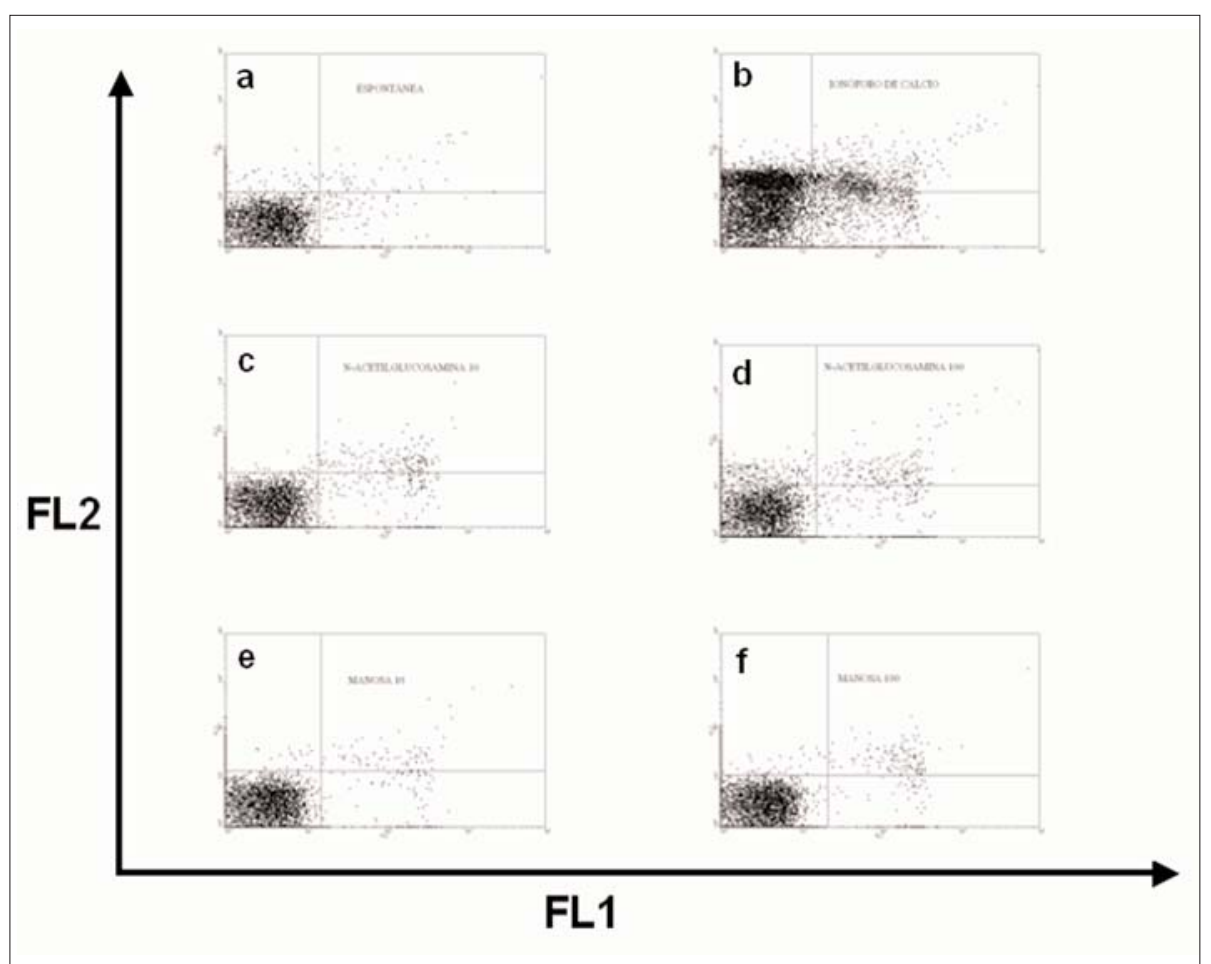

FIGURA 4. Diagrama de puntos de espermatozoides marcados con anticuerpo anti CD46-FITC e yoduro de propidio. Cada panel está dividido en cuatro cuadrantes, representando diferentes subpoblaciones de espermatozoides acorde con su estado acrosomal y viabilidad: en el cuadrante inferior izquierdo se localizan las células vivas y con acrosoma intacto, el cuadrante superior izquierdo representa los espermatozoides permeables al ioduro (células muertas) y con acrosoma intacto, en el cuadrante inferior derecho se ubican los espermatozoides vivos reaccionados y el cuadrante superior derecho representa los espermatozoides permeables al yoduro pero que han sufrido reacción acrosomal; (a) reacción acrosomal espontánea, (b) estimulo con ionóforo de calcio A23187, ( $c$ y d) estimulo con $\mathrm{N}$-acetilglucosamina a 10 y $100 \mathrm{mg} / \mathrm{ml}$, respectivamente y (e y f) estimulo con manosa a 10 y $100 \mathrm{mg} / \mathrm{ml}$, respectivamente.

aunque es importante recalcar que en estos estudios los espermatozoides fueron tratados con los estímulos después de la separación, sin ser capacitados previamente, y el proceso de capacitación permite un incremento tanto de los receptores de fucosa y manosa $^{19,28}$ como de los receptores de $\mathrm{N}$-acetilglucosamina según nuestra experimentación.

El aumento de los receptores de manosa y de $\mathrm{N}$-acetilglucosamina después de la capacitación están soportados por un estudio en el cual al preincubar los espermatozoides humanos con los respectivos monosacáridos se disminuye la capacidad de unión de los espermatozoides a los oocitos. Estos autores usaron el ensayo de hemizona para evaluar la capacidad de unión; sin embargo aunque describen que hay una disminución en la capacidad de unión, no encuentran

ridos inducen la reacción acrosomal, sino que éste es un fenómeno complejo que involucra muchas vías y procesos.

De otro lado, en la zona pelúcida del humano $^{25}$ y del hámster ${ }^{38,39}$ hay residuos de $\mathrm{N}$-acetilglucosamina, y como se observa en nuestros resultados, la capacidad de inducción de la reacción acrosomal por incubación con la N-acetilglucosamina aumentó después de la capacitación, lo que sugiere que el receptor para esta molécula podría ser también dependiente de cambios en la membrana del espermatozoide, como ha sido reportado para el receptor de manosa ${ }^{30}$ (Fig. 2). Sin embargo, en algunos estudios ${ }^{19,28}$ el tratamiento de espermatozoides con $\mathrm{N}$-acetilglucosamina, manosa y fucosa antes de la coincubación con los oocitos no produjo ningún efecto aparente, efecto en la inducción de la reacción acrosomal ${ }^{18}$. Adicionalmente la presencia de monosacáridos como manosa y $\mathrm{N}$-acetilglucosamina fue observada después de realizar tratamientos enzimáticos de las membranas plasmáticas ${ }^{40}$, lo que hace pensar que los espermatozoides pueden tener ligandos para receptores presentes en la zona pelúcida, haciendo de este proceso de interacción intergamentos un proceso mucho más complejo.

Otro hallazgo importante de nuestro trabajo fue la diferencia en la inducción de la reacción acrosomal por citometría de flujo y por microscopía de fluorescencia, ya que con la primera no se observó inducción de la reacción acrosomal mientras que con la segunda sí se incrementó el porcentaje de espermatozoides reaccionados al estimular con los monosacáridos $(p<0,0001)$. 


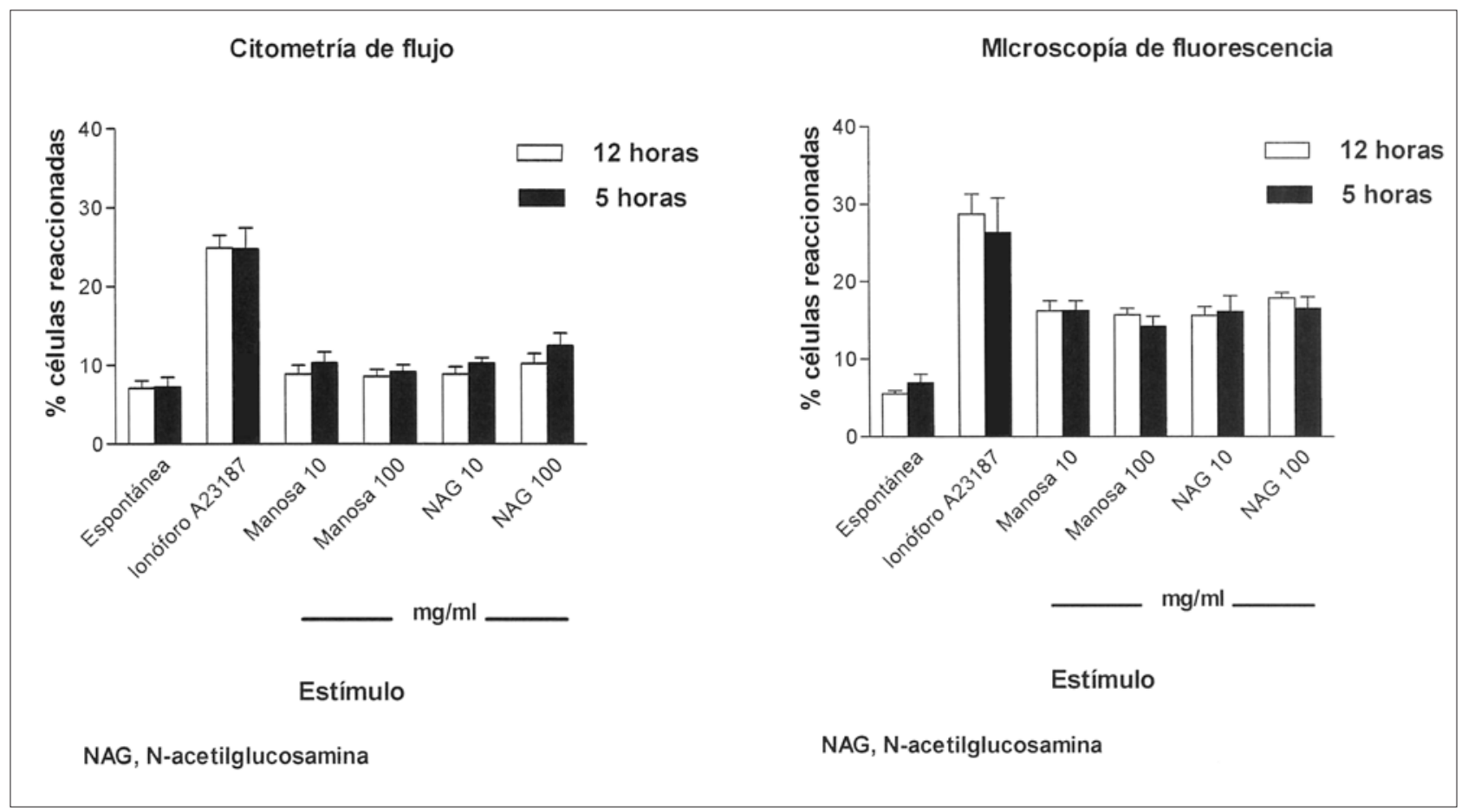

FIGURA 5. Comparación del porcentaje de inducción de la reacción acrosomal evaluada por citometría de flujo y microscopia de fluorescencia, después de 5 y 12 horas de capacitación.

Después de revisar algunos artículos ${ }^{41-43}$ podemos proponer que los monosacáridos manosa y $\mathrm{N}$-acetilglucosamina inducen reacción acrosomal parcial, ya que ésta es posible determinarla por microscopía de fluorescencia y no mediante citometría de flujo, que sólo permite cuantificar las células que presentan reacción acrosomal completa.

El término de reacción acrosomal parcial y completa se refiere al estado acrosomal de los espermatozoides: la reacción acrosomal parcial se define como la liberación parcial del contenido acrosomal o la pérdida parcial de la membrana acrosomal externa, y por reacción acrosomal completa se entiende la exposición de la membrana acrosomal interna y la pérdida completa del contenido acrosomal ${ }^{41-43}$. Una de las diferencias entre los métodos PSA-FITC y el anti-CD46FITC es que la PSA es una tinción negativa, porque colorea la membrana acrosomal externa o el contenido acrosomal y cuando se da la exocitosis, se evalúa la desaparición del marcaje. A diferencia de la PSA, el anti-CD-46 unido a un fluorocromo es una coloración positiva, es decir, que sólo los espermatozoides reaccionados son mar- cados; esta metodología sólo permite detectar el paso final de la reacción acrosomal cuando la membrana acrosomal interna es expuesta ${ }^{1}$, y allí se encuentra la proteína CD46.

Una de las preguntas que surge al plantear las dos clases de reacción acrosomal es ¿cuál es la que necesita el espermatozoide para poder fertilizar: reacción acrosomal parcial o completa?. Por la metodología usada en este trabajo en la cual no se usaron nunca oocitos, ni hubo interacción intergametos sólo podemos discutir lo reportado en la literatura. Desde los años 50 Austin y Bishop escribieron "la lisis de la zona pelúcida no ha sido aún demostrada, pero un pequeño agujero en la zona después del paso del espermatozoide podría probar la existencia"44; esto permitió detectar varias enzimas en el acrosoma como la acrosina, la arilsulfatasa y la $\beta$-N-acetilhexasaminidasa pero su papel en dicho proceso sigue siendo aun motivo de estudio, ya que se reconoce que la reacción acrosomal es un prerrequisito para que el espermatozoide pueda penetrar a través de la zona pelúcida. Sin embargo, la reacción acrosomal no es sólo la liberación de enzimas sino que además es el proceso por el cual el 
espermatozoide sufre cambios en la parte apical de la cabeza dándole unas características de terminación en punta que favorecería la penetración porque le permite abrirse camino por si sólo a través de la ZP2 y la ZP1, por lo tanto después de la liberación enzimática se da una segunda fase de unión del espermatozoide a la zona pelúcida la cual no es dependiente de enzimas ${ }^{45}$.

A partir de nuestros resultados, y en concordancia con Crosby y cols ${ }^{45}$, podríamos proponer que el espermatozoide al entrar en contacto con los monosacáridos de la zona pelúcida inicia el proceso de reacción acrosomal parcial para poder empezar a atravesar la ZP3, sufriendo los cambios en la estructura de la cabeza, los cuales le permitirán, en compañía del resto de contenido acrosomal, penetrar completamente la zona pelúcida.

\section{REFERENCIAS}

1. Patrat C, Serres C, Jouannet P. The acrosome reaction in human spermatozoa. Biol Cell 2000;92:255-266.

2. Topfer_Petersen E. Carbohydrate-based interactions on the route of a spermatozoon to fertilization. Hum Reprod Update 1999;5:314-329.

3. Bleil JD, Wassarman PM. Structure and function of the zona pellucida: identification and characterization of the proteins of the mouse oocyte's zona pellucida. Dev Biol 1980;76:185-202.

4. Leyton L, LeGuen P, Bunch D, Saling PM. Regulation of mouse gamete interaction by a sperm tyrosine kinase. Proc Natl Acad Sci U S A 1992;89:11692-11695.

5. Tomes CN, Roggero CM, De Blas G, Saling PM, Mayorga LS. Requirement of protein tyrosine kinase and phosphatase activities for human sperm exocytosis. Dev Biol 2004; 265:399-415.

6. Nixon B, Lu Q, Wassler MJ, Foote CI, Ensslin MA, Shur BD. Galactosyltransferase function during mammalian fertilization. Cells Tissues Organs 2001;168:46-57.

7. Haines N, Irvine KD. Functional analysis of Drosophila \{beta\} 1,4-N-Acetlygalactosaminyltransferases. Glycobiology 2004.

8. Cohen N, Wassarman PM. Association of egg zona pellucida glycoprotein mZP3 with sperm protein sp56 during fertilization in mice. Int J Dev Biol 2001;45:569-76.

9. Topfer-Petersen E, Romero A, Varela PF, et al. Spermadhesins: a new protein family. Facts, hypotheses and perspectives. Andrologia 1998;30:217-224.

10. Hardy DM, Garbers DL. A sperm membrane protein that binds in a species-specific manner to the egg extracellular matrix is homologous to von Willebrand factor. J Biol Chem 1995;270:26025-26028.

11. Lea IA, Sivashanmugam P, O'Rand MG. Zonadhesin: characterization, localization, and zona pellucida binding. Biol Reprod 2001;65:1691-1700.

12. Gabius HJ. The how and why of protein-carbohydrate interaction: a primer to the theoretical concept and a guide to application in drug design. Pharm Res 1998;15:23-30.
13. Villalobo A, Gabius H. Signaling pathways for transduction of the initial message of the glycocode into cellular responses. Acta Anat (Basel) 1998;161:110-129.

14. Solis D, Jimenez-Barbero J, Kaltner H, et al. Towards defining the role of glycans as hardware in information storage and transfer: basic principles, experimental approaches and recent progress. Cells Tissues Organs 2001;168:5-23.

15. Topfer-Petersen E, Petrounkina AM, Ekhlasi-Hundrieser M. Oocyte-sperm interactions. Anim Reprod Sci 2000;6061:653-662.

16. Tulsiani DR, Yoshida-Komiya H, Araki Y. Mammalian fertilization: a carbohydrate-mediated event. Biol Reprod 1997; 57:487-494.

17. Macek MB, Shur BD. Protein-carbohydrate complementarity in mammalian gamete recognition. Gamete Res 1988; 20:93-109.

18. Miranda PV, Gonzalez-Echeverria F, Marin-Briggiler CI, Brandelli A, Blaquier JA, Tezon JG. Glycosidic residues involved in human sperm-zona pellucida binding in vitro. Mol Hum Reprod 1997;3:399-404.

19. Mori K, Daitoh $T$, Irahara $M$, Kamada $M$, Aono $T$. Significance of D-mannose as a sperm receptor site on the zona pellucida in human fertilization. Am J Obstet Gynecol 1989;161:207-211.

20. Mori K, Daitoh T, Kamada M, et al. Blocking of human fertilization by carbohydrates. Hum Reprod 1993;8:17291732 .

21. Benoff S, Cooper GW, Hurley I, et al. Human sperm fertilizing potential in vitro is correlated with differential expression of a head-specific mannose-ligand receptor. Fertil Steril 1993;59:854-862.

22. Chen JS, Doncel GF, Alvarez C, Acosta AA. Expression of mannose-binding sites on human spermatozoa and their role in sperm-zona pellucida binding. J Androl 1995;16: 55-63.

23. Brandelli A, Miranda PV, Tezon JG. Voltage-dependent calcium channels and Gi regulatory protein mediate the human sperm acrosomal exocytosis induced by N-acetylglucosaminyl/mannosyl neoglycoproteins. J Androl 1996; 17:522-529.

24. Loeser CR, Tulsiani DR. The role of carbohydrates in the induction of the acrosome reaction in mouse spermatozoa. Biol Reprod 1999;60:94-101.

25. Brandelli A, Miranda PV, Tezon JG. Participation of glycosylated residues in the human sperm acrosome reaction: possible role of $\mathrm{N}$-acetylglucosaminidase. Biochim Biophys Acta 1994;1220:299-304.

26. Gabriele A, D’Andrea G, Cordeschi G, et al. Carbohydrate binding activity in human spermatozoa: localization, specificity, and involvement in sperm-egg fusion. Mol Hum Reprod 1998;4:543-553.

27. Oehninger S, Acosta A, Hodgen GD. Antagonistic and agonistic properties of saccharide moieties in the hemizona assay. Fertil Steril 1990;53:143-149.

28. Oehninger S, Clark GF, Acosta AA, Hodgen GD. Nature of the inhibitory effect of complex saccharide moieties on the tight binding of human spermatozoa to the human zona pellucida. Fertil Steril 1991;55:165-169.

29. Oehninger S, Clark GF, Fulgham D, et al. Effect of fucoidin on human sperm-zona pellucida interactions. J Androl 1992; 13:519-525.

30. Benoff S, Hurley IR, Mandel FS, Cooper GW, Hershlag A. Induction of the human sperm acrosome reaction with mannose-containing neoglycoprotein ligands. Mol Hum Reprod 1997;3:827-837. 
31. Yanagimachi R. Fertilization in Mammalian. In: Knobil E NJ, ed. The Physiology of Reproduction. New York: Raven Press Ltd., 1994:189-318.

32. Brandelli A, Miranda PV, Anon-Vazquez MG, et al. A new predictive test for in-vitro fertilization based on the induction of sperm acrosome reaction by $\mathrm{N}$-acetylglucosamineneoglycoprotein. Hum Reprod 1995;10:1751-1756.

33. Bendahmane M, Tulsiani DR. Capacitated acrosome-intact mouse spermatozoa bind to Sepharose beads coated with functional neoglycoproteins. Arch Biochem Biophys 2003;415:203-212.

34. Bendahmane M, Zeng HT, Tulsiani DR. Assessment of acrosomal status in rat spermatozoa: studies on carbohydrate and non-carbohydrate agonists. Arch Biochem Biophys 2002;404:38-47.

35. Tea N, T., Jondet M, Scholler R. migration-gravity sedimentation' method for collecting motile spermatozoa from human semen. In: Harrison R, Bonnar J, Thompson W, eds. In Vitro Fertilization, Embryo Transfer and Early Pregnancy: Lancaster: MTP Press Ltd, 1984:117-120.

36. WHO. WHO Laboratory Manual for the Examination of Human Semen and Sperm-Cervical Mucus Interaction. Cambridge: Cambridge University Press, 1999.

37. Cardona W, Berdugo J, De los Rios J, Cadavid A. Sperm functional evaluation in fertile men. En preparación. 2005.

38. Ahuja KK. Fertilization studies in the hamster. The role of cell-surface carbohydrates. Exp Cell Res 1982;140:353362.

39. Zitta K, Wertheimer E, Miranda PV. Analysis of the participation of $\mathrm{N}$-acetylglucosamine in the different steps of sperm-zona pellucida interaction in hamster. Mol Hum Reprod 2004;10:925-33.
40. Tortorella H, Konrath RA, Mazzini MN, Brandelli A. Proteolytic release and partial characterization of human sperm-surface glycopeptides. Braz J Med Biol Res 1997;30:387-93.

41. Jaiswal BS, Cohen-Dayag A, Tur-Kaspa I, Eisenbach M. Sperm capacitation is, after all, a prerequisite for both partial and complete acrosome reaction. FEBS Lett 1998;427:309-13.

42. Jaiswal BS, Eisenbach M, Tur-Kaspa I. Detection of partial and complete acrosome reaction in human spermatozoa: which inducers and probes to use? Mol Hum Reprod 1999;5:214-9.

43. Kohn FM, Mack SR, Schill WB, Zaneveld LJ. Detection of human sperm acrosome reaction: comparison between methods using double staining, Pisum sativum agglutinin, concanavalin A and transmission electron microscopy. Hum Reprod 1997;12:714-21.

44. Bedford JM. Mammalian fertilization misread? Sperm penetration of the eutherian zona pellucida is unlikely to be a lytic event. Biol Reprod 1998;59:1275-87.

45. Crosby JA, Jones R, Barros C, Carvallo P. Characterization of the functional domains of boar acrosin involved in nonenzymatic binding to homologous zona pellucida glycoproteins. Mol Reprod Dev 1998;49:426-34.

W. Cardona-Maya

Calle 62 \# 52-59,

1226 Medellín (Antioquia) Colombia

e-mail: wdcmaya@yahoo.com

(Trabajo recibido el 6 mayo de 2005) 